\title{
Emergence of KPC-producing Klebsiella pneumoniae in Italy
}

\author{
Carla Fontana ${ }^{1,2^{*}}$, Marco Favaro ${ }^{1}$, Loredana Sarmati ${ }^{4}$, Silvia Natoli ${ }^{3}$, Anna Altieri ${ }^{2}$, Maria C Bossa ${ }^{2}$, Silvia Minelli ${ }^{2}$, \\ Francesca Leonardis ${ }^{3}$, Cartesio Favalli, ${ }^{1,2}$
}

\begin{abstract}
Background: The emergence of KPC-producing K. pneumoniae has now become a global concern. KPC betalactamases are plasmid-borne and, like extended spectrum beta lactamases (ESBLs), can accumulate and transfer resistance determinants to other classes of antibiotics. Therefore, infection control guidelines on early identification and control of the spread of organisms carrying these resistant determinants are needed.

Findings: Klebsiella pneumoniae carbapenemase (KPC) was detected in two isolates of carbapenem-resistant $K$. pneumoniae obtained from patients at an Italian teaching hospital. The first strain was isolated from a culture drawn from a central venous device (CVC) in a patient with Crohn's disease who was admitted to a gastroenterology ward. The second was isolated from a urine sample collected from an indwelling urinary catheter in an intensive care unit (ICU) patient with a subdural haematoma. The patients had not travelled abroad. Both isolates were resistant to all $\beta$-lactams and were susceptible to imipenem and meropenem but resistant to ertapenem. Isolates also showed resistance to other classes of non- $\beta$-lactam antibiotics, such as quinolones, aminoglycosides (with the exception for amikacin), trimethoprim-sulfamethoxazole (TMP-SMX) and nitrofurantoin. They were determined to contain the plasmid encoding the carbapenemase gene bla-KPC and were also positive in the Hodge test.
\end{abstract}

Conclusions: This is the second report of KPC-producing isolates in Italy, but the first concerning KPC type 2 gene, and it may have important implications for controlling the transmission of microorganisms resistant to antibiotics.

\section{Findings}

Klebsiella pneumoniae carbapenemase (KPC) is a molecular class A serine $\beta$-lactamase belonging to functional group 2f [1]. KPC was first reported in 2001 after its discovery in a K. pneumoniae clinical isolate collected in North Carolina during the ICARE (Intensive Care Antimicrobial Resistance Epidemiology) surveillance study [1-3]. The first detection of KPC-2 on a plasmid in $P$. aeruginosa was reported; this represents a disturbing development in the spread of these carbapenemases $[2,4]$. Although the KPC $\beta$-lactamases are predominantly found in K. pneumoniae, there have been reports of these enzymes in Enterobacter spp. and in Salmonella spp and in other genera of the Enterobacteriaceae family [5-10].

\footnotetext{
* Correspondence: carla.fontana@uniroma2.it

${ }^{1}$ Department of Experimental Medicine and Biochemical Sciences, "Tor Vergata" University of Rome - Via Montpellier 1, Rome, 00133, Italy
}

KPC-positive strains are typically resistant to the penicillins, extended-spectrum cephalosporins, and aztreonam, but the MICs of these drugs and of the carbapenems are reduced in the presence of clavulanic acid due to enzyme inhibition [2]. Isolates that acquire this enzyme are usually resistant to several other classes of antimicrobial agents used as treatment options. Laboratory identification of KPC-producing clinical isolates will be critical for limiting the spread of this resistance mechanism. The failure of automated susceptibility testing systems to detect KPC-mediated resistance was previously noted, particularly if ertapenem, which was determined to be more susceptible to the idrolytic activity of KPC carbanemase, was not tested [11]. After the rapid expansion of the KPC class of carbapenemases along the east coast of the United States, reports from across the world began to appear. A report from France in 2005 documented KPC-2 in a K. pneumoniae strain from a patient who had been in New York for medical
C Biomed Central 
treatment [12]. KPC-producing organisms have continued to spread over time and have now been reported in 27 states in the United States and in many countries around the world, including China, Colombia, Brazil, Israel, Canada, France, the Republic of Ireland, Greece (where infection caused by KPC is mainly due to hyperepidemic clone) and more recently in Italy [8-19]. We report, in this paper, the first two cases of KPC2 producing $K$. pneumoniae in Italy. Our finding confirms that KPC producing isolates are a global concern and that every effort should be made from the laboratory to early identification of these phenotypes.

\section{Case Presentation \\ Case 1}

A 48-year-old man was admitted to Tor Vergata University hospital in February 2009). He had suffered from Crohn's disease since 1990, with prolonged illness exacerbation periods. He received numerous treatments and had recently decided to treat himself with steroids. A colonoscopy was performed to remove a colic polyp, but an intra-abdominal perforation complicated the exam. A stercoraceous peritonitis developed and the patient urgently underwent intestinal resection and splenectomy. Seven days later, an ileal necrosis of the colostomy tract appeared and he underwent a resection of ileum. A few days later, he developed a fever and leucocitosis, and a Computed Tomography (CT) scan revealed an abdominal abscess that was drained. Different bacterial isolates were obtained from the blood and abscess drainage cultures (Enterococcus faecalis, Escherichia coli, Candida albicans, Klebsiella pneumoniae and Acineobacter baumannii), K. pneumoniae was cultured from the tip of a CVC (which was confirmed as KPC producing in two days). Numerous antibiotic treatments were used. The initial drug regimen consisted of linezolid, amikacin, caspofungin, piperacillin-tazobactam, metronidazole and then, after the identification of a KPC-producing $K$. pneumoniae (due to the positivity of the Hodge's test), the antibiotic therapy was shifted to an association of tigecycline $(100 \mathrm{mg}$ for the first dose and then $50 \mathrm{mg}$ every $12 \mathrm{~h}$ ) and amikacin ( $1 \mathrm{~g}$ once a day) for 9 days. At the time of multi-drug resistant $A$. baumannii isolation, amikacin was suspended and tigecycline continued in combination with colistin (2.000.000 units every $8 \mathrm{~h}$ ). The association of tigecycline and colistin was suspended after 16 days. The patient was discharged without fever, abdominal drainage was removed when nothing further was picked up.

\section{Case 2}

A 79-year-old woman with an intra-cerebral haemorrhage was admitted to the neurosurgical department of Tor Vergata University hospital in March, 2009. She had lost consciousness following a sudden headache and subsequent head trauma. The patient was operated on immediately and was later admitted to the ICU. Her past history revealed chronic atrial fibrillation, so she was administered dicumarol. Moreover, in the past she had suffered from recurrent urinary tract infections treated by her family doctor with ciprofloxacin. On admission, the patient was intubated and a urinary catheter was inserted, a central intravenous catheter was placed in her subclavian vein, and an arterial line was also inserted. She started a course of therapy with piperacillin/tazobactam (4.5 g/i.v. q6h) for the treatment of aspiration pneumonia due to $P$. aeruginosa. On the ninth day, the patient's temperature increased to $39^{\circ} \mathrm{C}$ and she experienced chills. Her white blood count and $C$ reactive protein also increased $\left(11.300 \mathrm{~mm}^{3}\right.$ and $102 \mathrm{mg} / \mathrm{L}$, respectively). Several blood cultures (from the CVC or from the peripheral vein), urine and bronchial secretions (BAS) were collected for cultures. An empirical treatment was started by adding teicoplanin $(600 \mathrm{mg}$ i.v. q24 h) and levofloxacin (500 mg i.v. q12h) to the previous antibiotic therapy. The blood cultures from the CVC resulted positive for Proteus mirabilis and Enterococcus faecalis. The culture of BAS evidenced the same $P$. aeruginosa previously isolated, while the culture of urines yielded $10^{6} \mathrm{CFU} / \mathrm{mL}$ of $K$. pneumoniae KPC-producing (initially confirmed by the Hodge's test). The previous antibiotic treatment was altered to amikacin (1,5 g i.v. q24h), while teicoplanin was maintained at the same dose. In the meantime, CVC was removed. After eight days, there was a second episode of fever. The amikacin treatment was interrupted and a tigecycline regimen was initiated (100 $\mathrm{mg}$ for the first dose and then $50 \mathrm{mg}$ q12h). Blood and urine samples were collected again and a single blood culture was positive for $P$. aeruginosa. After ten days of treatment with meropenem, the infectious event clinically resolved, but the patient's conditions slowly worsened. After 27 days of hospitalization, the patient died due to the progression of her brain damage.

\section{Methods}

Isolates were tested for susceptibility using Vitek2 (bioMérieux, Durham, NC), using AST GN13 and AST GN074, as well as the AST N022 card. Susceptibility was also confirmed for meropenem, imipenem, ertapenem, colistin and amikacin using Etest (bioMérieux Italia; Firenze, Italy) according to the manufacturer's recommendations and incubated for 18 hours at $35^{\circ} \mathrm{C}$ in ambient air. MICs were interpreted according to the Clinical Laboratory Standards Institute (CLSI) guidelines [20]. MIC for colistin were interpreted according to the EUCAST breakpoints available at the web site http:// www.srga.org/eucastwt/MICTAB/index.html. Isolates 
were also tested using the modified Hodge test [21]. To exclude the possibility of a metallo-beta-lactamases (MBL) production a synergy test using imipenem and EDTA discs was used, the test was performed according to CLSI [20,22]

Analysis of $\beta$-lactamase genes (including bla $a_{\mathrm{KPC}}$ $b l a_{\mathrm{TEM}} b l a_{\mathrm{OXA}}$, and $\left.b l a_{\mathrm{SHV}}\right)$, CTX-M type, VIM type, IMP type as well as blaAmpC were preformed as previously reported [13,23-26].

Amplified products of the expected size were confirmed as KPC by sequencing. For typing of the bla $a_{\mathrm{KPC}}$ gene, overlapping PCR reactions were performed using the following primer pairs: F-5'CGGAACCATTCGCTAAACTC3' and R-3'GGCGGCGTTATCACTGTATT5' primers; F-5'CGCCGTGCAATACAGTGATA3' and R-3'CGTTGACGCCCAATCC5' primers [13].

Amplification products were purified using the Montage PCR Centrifugal Filter Device (Millipore Corporation, Billerica, MA), and sequencing was performed by Big Dye Terminators V1.1 (Applied Biosystems, Foster City, CA) and migrated with an automated sequencer (ABI Prism 310; Applied Biosystems). Sequences were aligned and compared using the National Center for Biotechnology Information database http://www.ncbi. nlm.nih.gov/.

\section{Results}

Isolates from case 1 and case 2 were both multi-drug resistant, being resistant to all $\beta$-lactam, quinoloni and aminoglycosides (with the exception of amikacin), TMPSMX, and nitrofurantoin, and were susceptible to tigecycline and colistin (see Table 1). The behaviour versus carbapenemic compounds was typical for KPC isolates: both isolates were susceptible to imipenem (MIC = $1 \mu \mathrm{g} / \mathrm{L})$ and meropenem $(\mathrm{MIC}=2 \mu \mathrm{g} / \mathrm{L})$ and resistant to ertapenem $(\mathrm{MIC}=8 \mu \mathrm{g} / \mathrm{L})$. Screening with ertapenem indicated the possibility of a KPC, which was confirmed by the modified Hodge test. While the MBL test was negative. Analysis of $\beta$-lactamase genes by PCR and sequencing revealed the presence of blaKPC-2, and blaSHV-1, while genes encoding other enzymes blaTEM, blaOXA, CTX-M type, VIM type, IMP type as well as blaAmpC were not detected.

Both patients improved clinically with tigecycline treatment, even if for the second patient a septic event due to $P$. aeruginosa (successive to $K$. pneumoniae infections) needed to be treated with meropenem.

\section{Conclusion}

This is the second documented appearance of a class A carbapenemase-producing isolate of $K$. pneumoniae in Italy, harbouring KPC type 2 gene, that was not associated with travel abroad. KPC $\beta$-lactamases (KPC 1-7) confer decreased susceptibility or resistance to all $\beta$-lactams [14,27]. The isolate showed reduced susceptibility or resistance to four different antibiotics, limiting the therapeutic options to polymixin, amikacin, and tigecycline [13]. At present, these are the second published cases of KPC-producing K. pneumoniae infections in Italy, after that published by Giani et al [19]. The dissemination of KPC-producing organisms is no longer restricted to the United States and has emerged as a global concern, aided by the recently described mobile genetic element Tn 4401, which carries the KPC genes [28]. Carbapenemase-producing pathogens cause infections that are difficult to treat and have high mortality rates due to their appearance in multidrug-resistant pathogens such as $K$. pneumoniae, P. aeruginosa, and Acinetobacter spp [2]. The first descriptions of these enzymes as species-specific chromosomal carbapenemases have more recently been followed by the appearance of carbapenemase genes that are easily transferred on mobile elements between species. While considered by some to be relatively rare, reports of their occurrence in outbreak settings have steadily increased $[2,13,14]$. Detecting their entry into the hospital environment is the first step that clinical microbiologists can take to address this problem. Care in detection is needed, because high carbapenem MICs are not always evident and the phenotype could be confused with that described by Cagnacci et al $[11,22,29]$. Therefore, it is essential that the initial complete screening of the isolate includes Hodge's test as well as MBL test. Evaluation of effective antibiotic options and rigorous infection control measures will help in the fight against carbapenemase-producing organisms [2]. Regardless of the geographic location, microbiology laboratories in Italy need to ensure that they have methods in place for the accurate detection of KPC-producing organisms and must constantly be aware of their potential to spread.

Table 1 Susceptibility of KPC-producing Klebsiella pneumoniae isolates

\begin{tabular}{ccccccccccccccccc}
\hline \multicolumn{110}{c}{ Isolates } & AMP & AMC & PIP & PTZ & CAZ & CTX & FEP & IPM & MEM & EPT & AMK & CIP & TGC & CS & TMP-SMX & NT \\
\hline Case 1 isolate & 32 & 32 & 128 & 128 & 16 & 64 & 16 & 1 & 2 & 8 & 2 & 8 & 0.5 & 0.5 & 320 & 512 \\
\hline Case 2 isolate & 32 & 32 & 128 & 128 & 16 & 64 & 16 & 1 & 2 & 8 & 2 & 8 & 0.5 & 0.5 & 320 & 512 \\
\hline
\end{tabular}

Abbreviations: AMP, ampicillin, AMC, amoxicillin-clavulanate; PIP, piperacillin, PTZ, piperacillin-tazobactam; CAZ, ceftazidime; CTX, cefotaxime, FEP, cefepime; IPM, imipenem; MEM, meropenem; EPT, ertapenem; AMK, amikacin; CIP, ciprofloxacin; TGC, tigecycline; CS, colistin; TMP-SMX, trimethoprim-sulfamethoxazole; NT, nitrofurantoin. 


\section{List of abbreviations used}

KPC: Klebsiella pneumoniae carbapenemase; CVC: Central venousdevice; ICARE: Intensive Care Antimicrobial Resistance Epidemiology; ICU: Intensive care unit; TMP-SMX: Trimethoprim-sulfamethoxazole; CT: Computed Tomography; BAS: Bronchial secretions; CLSI: Clinical Laboratory Standards Institute; MBL: Metallobeta-lactamases.

\section{Consent}

Given the impossibility of finding the first patient (lost to follow up) and the death of the second, the ethics committee of the hospital gave approval for anonymous publication of these clinical cases (n 17/08 English version 04/2009).

\section{Acknowledgements}

We thank Francesca Capalbo, Oriana Cicchetti, Marco Pelliccioni, Alessandro Mauti, and Janet Dianetti for their technical assistance.

\section{Author details \\ 'Department of Experimental Medicine and Biochemical Sciences, "Tor Vergata" University of Rome - Via Montpellier 1, Rome, 00133, Italy. ${ }^{2}$ Clinical Microbiology Laboratories, Polyclinic of Tor Vergata, Viale Oxford 81, Rome, 00133, Italy. ${ }^{3}$ Department of Surgery, Intensive Care Unit, Polyclinic Tor Vergata, Viale Oxford 81, Rome, 00133, Italy. ${ }^{4}$ Infectious Diseases, Department of Public Health, Polyclinic of Tor Vergata, Viale Oxford 81, Rome, 00133, Italy.}

\section{Authors' contributions}

CF and LS, SN and MF contributed to the conception of the study, in data analysis and are also involved in drafting the manuscript. FL and CsF contributed to the review of the study and data analysis. AA, MCB and SM contributed in acquisition and interpretation of data. All authors approved the final version of the manuscript.

\section{Competing interests}

All authors declare no financial or personal relationships with other people or organizations that could inappropriately have influenced (bias) their work.

Received: 3 September 2009 Accepted: 23 February 2010

Published: 23 February 2010

\section{References}

1. Wolter DJ, Kurpiel PM, Woodford N, Palepou MF, Goering RV, Hanson ND: Phenotypic and enzymatic comparative analysis of the novel KPC variant KPC-5 and its evolutionary variants, KPC-2 and KPC-4. Antimicrob Agents Chemother 2009, 53:557-62.

2. Queenan AM, Bush K: Carbapenemases: the versatile beta-lactamases. Clin Microbiol Rev 2007, 20:440-58.

3. Yigit H, Queenan AM, Anderson GJ, Domenech-Sanchez A, Biddle JW, Steward CD, Alberti S, Bush K, Tenover FC: Novel carbapenem-hydrolyzing beta-lactamase, KPC-1, from a carbapenem-resistant strain of Klebsiella pneumoniae. Antimicrob Agents Chemother 2001, 45:1151-1161.

4. Villegas MV, Lolans K, Correa A, Kattan JN, Lopez JA, Quinn JP, the Colombian Nosocomial Resistance Study Group: First identification of Pseudomonas aeruginosa isolates producing a KPC-type carbapenem-hydrolyzing betalactamase. Antimicrob Agents Chemother 2007, 51:1553-1555.

5. Bratu S, Landman D, Alam M, Tolentino E, Quale J: Detection of KPC carbapenem-hydrolyzing enzymes in Enterobacter spp. From Brooklyn, New York. Antimicrob Agents Chemother 2005, 49:776-778.

6. Hossain A, Ferraro MJ, Pino RM, Dew RBI II, Moland ES, Lockhart TJ, Thomson KS, Goering RV, Hanson ND: Plasmid mediated carbapenemhydrolyzing enzyme KPC-2 in an Enterobacter sp. Antimicrob Agents Chemother 2004, 48:4438-4440.
7. Miriagou V, Tzouvelekis LS, Rossiter S, Tzelepi E, Angulo FJ, Whichard JM: Imipenem resistance in a Salmonella clinical strain due to plasmidmediated class A carbapenemase KPC-2. Antimicrob Agents Chemother 2003, 47:1297-1300.

8. Cai JC, Zhou HW, Zhang R, Chen GX: Emergence of Serratia marcescens, Klebsiella pneumoniae, and Escherichia coli isolates possessing the plasmid-mediated carbapenem-hydrolyzing beta-lactamase KPC-2 in intensive care units of a Chinese hospital. Antimicrob Agents Chemother 2008, 52:2014-2018.

9. Villegas MV, Lolans K, Correa A, Suarez CJ, Lopez JA, Vallejo M, Quinn JP: First detection of the plasmid-mediated class A carbapenemase KPC-2 in clinical isolates of Klebsiella pneumoniae from South America. Antimicrob Agents Chemother 2006, 50:2880-2882.

10. Rasheed JK, Biddle JW, Anderson KF, Washer L, Chenoweth C, Perrin J, Newton DW, Patel JB: Detection of the Klebsiella pneumoniae carbapenemase type 2 carbapenem-hydrolyzing enzyme in clinical isolates of Citrobacter freundii and Klebsiella oxytoca carrying a common plasmid. J Clin Microbiol 2008, 46:2066-2069.

11. Anderson KF, Lonsway DR, Rasheed JK, Biddle J, Jensen B, McDougal LK, Carey RB, Thompson A, Stocker S, Limbago B, Patel JB: Evaluation of methods to identify the Klebsiella pneumoniae carbapenemase in Enterobacteriaceae. J Clin Microbiol 2007, 45:2723-5.

12. Naas T, Nordman P, Vedel G, Poyart C: Plasmid-mediated carbapenemhydrolyzing beta-lactamase KPC in a Klebsiella pneumoniae isolate from France. Antimicrob Agents Chemother 2005, 49:4423-4.

13. Goldfarb D, Harvey SB, Jessamine K, Jessamine P, Toye B, Desjardins M: Detection of plasmid mediated KPC-Producing Klebsiella pneumoniae in Ottawa, Canada: Evidence of Intra-Hospital Transmission. J Clin Microbiol 2009, 47(6):1920-2.

14. Roche C, Cotter M, O Connell N, Crowley B: First identification of class A carbapenemase-producing Klebsiella pneumoniae in the Republic of Ireland. Euro Surveill 2009, 2(14(13)), pii: 19163

15. Kitchel B, Rasheed JK, Srinivasan A, McDougal LK, Patel JB: Molecular epidemiology of KPC-producing Klebsiella pneumoniae in the United States. Program and abstracts of the 48th Annual ICAAC/IDSA 46th Annual Meeting: A Joint Meeting of the American Society for Microbiology and the Infectious Diseases Society of America; October 25-28, 2008; Washington, DC.

16. Peirano G, Seki LM, Val Passos VL, Pinto MC, Guerra LR, Asensi MD: Carbapenem-hydrolysing beta-lactamase KPC-2 in Klebsiella pneumoniae isolated in Rio de Janeiro, Brazil. J Antimicrob Chemother 2009, 63:265-268.

17. Leavitt A, Navon-Venezia S, Chmelnitsky I, Schwaber MJ, Carmeli Y: Emergence of KPC-2 and KPC-3 in carbapenem-resistant Klebsiella pneumoniae strains in an Israeli hospital. Antimicrob Agents Chemother 2007, 51:3026-3029.

18. Giakoupi P, Maltezou H, Polemis M, Pappa O, Saroglou G, Vatopoulos A the Greek System for the Surveillance of Antimicrobial Resistance: KPC-2producing Klebsiella pneumoniae infections in Greek hospitals are mainly due to a hyperepidemic clone. Euro Surveill 2009, 28:19218.

19. Giani T, D'Andrea MM, Pecile P, Borgianni L, Nicoletti P, Tonelli F, Bartoloni A, Rossolini GM: Emergence in Italy of Klebsiella pneumoniae sequence type 258 producing KPC-3 Carbapenemase. J Clin Microbiol 2009, 47:3793-4.

20. CLSI (Performance Standards for antimicrobial susceptibility testing): Clinical and Laboratory Standards Institute: Performance standards for antimicrobial susceptibility testing. Nineteenth informational supplement, M100-S19 Clinical and Laboratory Standards Institute, Wayne, PA 2009.

21. Lee K, Chong Y, Shin HB, Kim YA, Yong D, Yum JH: Modified Hodge and EDTA-disk synergy tests to screen metallo-beta-lactamase- producing strains of Pseudomonas and Acinetobacter species. Clin Microbiol Infect 2001, 7:88-91.

22. Cagnacci S, Gualco L, Roveta S, Mannelli S, Borgianni L, Docquier JD, Dodi F, Centanaro M, Debbia E, Marchese A, Rossolini GM: Bloodstream infections caused by multidrug-resistant Klebsiella pneumoniae producing the carbapenem-hydrolysing VIM-1 metallo-beta-lactamase: first Italian outbreak. J of Antimicrob Chemother 2008, 61:296-300.

23. Lomaestro BM, Tobin EH, Shang W, Gootz T: The Spread of Klebsiella pneumoniae Carbapenemase-Producing K. pneumoniae to Upstate New York. Clin Infect Dis 2006, 43:26-28.

24. Kaczmarek FM, Dib-Hajj F, Shang W, Gootz TD: High-level carbapenem resistance in a Klebsiella pneumoniae clinical isolate is due to the 
combination of bla(ACT-1) beta-lactamase production, porin OmpK35/36 insertional inactivation, and down-regulation of the phosphate transport porin phoe. Antimicrob Agents Chemother 2006, 50:3396-406.

25. Arpin $C$, Coulange $L$, Dubois V, André $C$, Fischer I, Fourmaux $S$, Grobost $F$, Jullin J, Dutilh B, Couture JF, Noury P, Lagrange I, Ducastaing A,

Doermann HP, Quentin C: Extended-spectrum-beta-lactamase-producing Enterobacteriaceae strains in various types of private health care centers. Antimicrob Agents Chemother 2007, 51:3440-4.

26. Perez-Perez FJ, Hanson ND: Detection of plasmid-mediated AmpC betalactamase genes in clinical isolates by using multiplex PCR. J Clin Microbiol 2002, 40:2153-2162

27. Poirel L, Pitout JD, Nordmann P: Carbapenemases: molecular diversity and clinical consequences. Future Microbiol 2007, 2:501-12.

28. Naas T, Cuzon G, Villegas MV, Lartigue MF, Quinn JP, Nordmann P: Genetic structures at the origin of acquisition of the beta-lactamase blakPC gene. Antimicrob Agents Chemother 2008, 52:1257-63.

29. Tenover FC, Kalsi RK, Williams PP, Carey RB, Stocker S, Lonsway D, Rasheed JK, Biddle JW, McGowan JE Jr, Hanna B: Carbapenem resistance in Klebsiella pneumoniae not detected by automated susceptibility testing. Emerg Infect Dis 2006, 12:1209-13.

doi:10.1186/1756-0500-3-40

Cite this article as: Fontana et al: Emergence of KPC-producing

Klebsiella pneumoniae in Italy. BMC Research Notes 2010 3:40.

\section{Submit your next manuscript to BioMed Central} and take full advantage of:

- Convenient online submission

- Thorough peer review

- No space constraints or color figure charges

- Immediate publication on acceptance

- Inclusion in PubMed, CAS, Scopus and Google Scholar

- Research which is freely available for redistribution

Submit your manuscript at www.biomedcentral.com/submit 